\title{
Intratympanic bupivacaine instillation for tinnitus treatment?
}

Joseph Eldor

Correspondence: csen_international@csen.com

Theoretical Medicine Institute, Jerusalem 9127302, Israel.

\begin{abstract}
Tinnitus is an early diagnostic sign of an inadvertent intravenous injection of bupivacaine epidural test dose. However, Bupivacaine can also relieve tinnitus. For example, a 57-year-old man undergoing facet and sacroiliac infiltration with lidocaine and bupivacaine experienced symptoms of systemic local anesthetic toxicity. He described significant perioral numbness. Shortly after this the patient noted that his long-standing and severe tinnitus was completely gone. Another study of a total of 50 young soldiers hospitalized for high frequency hearing loss and tinnitus following exposure to gun impulse noise was studied in order to ascertain the effects of two kinds of medical treatment. In a 17 subjects gangliosides were associated with subcutaneous infiltration of bupivacaine chlorhydrate (0.5\%). An improvement in hearing threshold (= greater than $20 \mathrm{~dB}$ at $4-8 \mathrm{kHz}$ ) and a consistent relief of tinnitus was respectively found in $66 \%$ of the treated subjects.
\end{abstract}

Keywords: Bupivacaine, tinnitus, intratympanic instillation

\section{Drug induced tinnitus}

It is well known among the lay public and medical/audiological professional communities that tinnitus may be elicited by a toxic drug overdose, eg., Quinine and Aspirin. Many medical treatments elicit a chemically induced tinnitus, i.e., iatrogenic. From a medical perspective, the possibility of a toxic effect of a drug prescribed for its pharmacologic and beneficial effect can occur. Classification of drug pharmaceuticals are primarily based on their principle actions. However, it is clear that no drug has only a single or specific effect. Chemically, extremely unlikely that any drug molecule will bind to only single species of molecular receptor. The number of potential receptors is large in every patient receiving pharmacological treatment. Even if the chemical structure of the drug allows it to bind to only one kind of receptor, the process biochemically controlled by such receptors, takes place in many different cell types and is therefore linked to many biochemical functions. The result is that both the patient and the physician probably experience and report more than one drug effect.

Drugs are selective rather than specific in their actions, because they bind more tightly to one than to a few types of other receptors. Furthermore, the receptors control discrete processes that have a specific effect. It is only because of their selectivity that drugs are useful in clinical medicine. However, the benificial effects of drugs must be routinely evaluated and correlated with their toxic effects. Such a correlation is in essence a monitoring sign, reflecting the border between the desired beneficial effect and the deleterious toxic side effect.

When studying our phamacologic tool-box we come across a broad variety of drugs which eventually elicit tinnitus. That is an accompaniment of drug overdoses; or unusually high drug retention in the body, eg., due to renal failure. Such drugs include the aminoglycoside antibiotics (Streptomycine, Gentamycine, Kanamycine, etc.)
Other antibiotics include Amphotericine, Chloramphenicol, Minocycline, Polymyxine B, Procain Penicilline, Sulfonamides, Vancomycine, etc.

Antineoplastic agents like Bleomycine, Cis-Platinum, Carboplatinum, Methotrexate, Nitrogen Mustard, Vinblastin, etc. are known for their possible side effect in the field of tinnitus.

The diuretics which may elicit tinnitus as a side effect include Acetazolamide, Bumetanide, Bendrofluazide, Clorotalidon, Diapamide, Etcrynic Acid, Furosemide, Hydrochlorthiazide, etc.

Another group of drugs which possibly may induce an unwanted tinnitus contains cardiac antiarhythmic drugs like Celiprolol, Quinidine, Flecainide, Lidocaine, Metoprolol, Propranolol, etc.

Psychopharmacological agents which may lead to tinnitus are many and include Amitryptiline, Benzodiazepine, Bupropion, Carbamzepine, Diclofensine, Doxepin, Desipramin, Fluoxetin, Imipramin, Lithium, Melitracen, Molindon, Paroxetin, Phenelzin, Protriptilin, Trazodon, Zimelidin, etc.

Several nonsteroidal anti-rheumatic agents may lead to tinnitus as Acetyl-Salicylate, Acemetacine, Benorilate, Benoxaprofen, Carprofen, Chloroquine, Diclofenac, Diflunisal, Fenoprofen, Feprazon, Ibuprofen, Indonretacine, Isoxicam, Ketoprofen, Naproxen, D-Penilicillamin, Phenylbutazon, Piroxicam, Proglumetacin, Proquazon, Sulindac, Tolmetin, Zomepirac, etc.

The synthetic partially modified glucocorticoide prednisolone may elicit tinnitus. Tinnitus is a possible side effect of local anesthetics such as Bupivacain, Tetracain, Lidocaine, etc.

Antimalarial agents are potent elicitors of tinnitus, eg., Quinine, Quinidine, Chloroquinine, Hydroxychloroquine, etc.

Other therapeutic substances with a potential risk to elicit tinnitus include Dihydroergotoxine, Doxylamine, oral contraceptives, Lidoflazine, etc.

Toxic substances long known to physicians to elicit tinnitus

(c) 2013 Joseph Eldor licensee Herbert Publications Ltd. This is an Open Access article distributed under the terms of Creative Commons Attribution License (http://creativecommons.org/licenses/by/3.0). This permits unrestricted use, distribution, and reproduction in any medium, provided the original work is properly cited. 
include alcohol, Arsenum, Lead, Caffeine, Marihuana, Nicotine, Mercury, etc.

This long and incoherent list of tinnitus eliciting drugs is so diverse that it is difficult to derive or establish one single paradigm or theory for the specific chemical interactions within the body as reflected by any and or all of these drugs. However, clinical experience in medicine has taught that tinnitus is as significant a symptom of human sickness as is pain and dizziness.

Furthermore, it is of interest that several of the above mentioned drugs are recommended for tinnitus treatment: Furosemide, Lidocaine, Amitryptiline, Carbamazapine, etc [1].

\section{K2p channels and tinnitus}

The second group of $\mathrm{K}+$ channels of interest is the two pore domain $\mathrm{K}+$ channels (K2p). Previously known as the delayed rectifier channels, these channels are believed to be responsible for the background or "leak" $\mathrm{K}+$ currents. In this setting they control the resting membrane potential. A blockade of these channels shifts the resting membrane potential towards spontaneous depolarisation. K2p channels are wide spread in the body. In the CNS they are mainly located in the thalamo-cortical and striatal neurons, where blockade leads to increased neuroexcitability. They are also present in high concentrations in the cerebral blood vessels, where blockade leads to vasoconstriction and decreased cerebral blood flow. K2p channels are also present in neurons of the auditory system, where blockade leads to tinnitus. $\mathrm{K} 2 \mathrm{p}$ channels are thought to mediate the stimulating effect of local anaesthetics on ventilation. They are located in the brainstem, where they modulate the respiratory response to carbon dioxide via chemo sensing of the $\mathrm{pH}$. They are also found in the carotid body, where they are expressed in the oxygen-sensing cells of the glomus body. K2p channels are sensitive to changes in $\mathrm{O} 2$ tension and extracellular $\mathrm{pH}$ and are potentiated by volatile anaesthetic [2].

\section{Tinnitus as bupivacaine toxicity}

Eldor et al., [3] published in 1988 that Tinnitus is an early diagnostic sign of an inadvertent intravenous injection of bupivacaine epidural test dose.

Bupivacaine, 30-50 mg, was administered intravenously to six normal human volunteers, over 10-15 min on two occasions, at least 2 weeks apart. All subjects experienced mild CNS toxicity, consisting of tinnitus, facial tingling, or subtle visual disturbances, associated with peak venous plasma concentrations of 0.81 to $2.7 \mathrm{micrograms} / \mathrm{ml}$ [4].

\section{Relief of tinnitus with bupivacaine}

Weinmeister [5] published in 2000 regarding Prolonged suppression of tinnitus after peripheral nerve block using bupivacaine and lidocaine. The local anesthetic lidocaine has been shown to suppress tinnitus, albeit very temporarily, when administered intravenously. Long-term suppression by local anesthetics has not been reported. Bupivacaine has not been studied. Weinmeister reported a case of prolonged (1-month) suppression of tinnitus following a peripheral nerve block performed with lidocaine and bupivacaine. A 57-year-old man undergoing facet and sacroiliac infiltration with lidocaine and bupivacaine experienced symptoms of systemic local anesthetic toxicity. He described significant perioral numbness. Shortly after this the patient noted that his long-standing and severe tinnitus was completely gone. Follow-up 1 month later revealed the tinnitus had not returned. Longer-term follow-up was not possible because the patient died. There are no reports regarding the use of bupivacaine for suppression of tinnitus. Although previous reports studying lidocaine for this purpose have shown only a brief effect, the use of bupivacaine or a combination of lidocaine and bupivacaine, as in this case, may represent a treatment for tinnitus that is worth further investigation. There currently is no effective long-term therapy for this debilitating problem.

\section{Bupivacaine and tinnitus}

Stewart et al., [6] compared the central nervous system (CNS) and cardiovascular effects of levobupivacaine and ropivacaine when given IV to healthy male volunteers $(\mathrm{n}=$ 14) in a double-blinded, randomized, crossover trial. Subjects received levobupivacaine $0.5 \%$ or ropivacaine $0.5 \%$ after a test infusion with lidocaine to become familiar with the early signs of CNS effects (eg., tinnitus, circumoral paresthesia, hypesthesia). The development of CNS symptoms was assessed at 1-min intervals and study drug administration was terminated when the first CNS symptoms were recognized. Thereafter, symptoms were recorded at 1-min intervals until symptom resolution. Hemodynamic variables were assessed by transthoracic electrical bioimpedance. Continuous 12-lead electrocardiogram monitoring was also performed. There was no significant difference between levobupivacaine and ropivacaine for: the mean time to the first onset of CNS symptoms $(P=0.870)$, mean total volume of study drug administered at the onset of the first CNS symptom $(P=0.595)$, stroke index $(P=0.678)$, cardiac index $(P=0.488)$, acceleration index $(P=0.697), P R$ interval $(P=0.213), Q R S$ duration $(P=$ 0.637), $Q T$ interval $(P=0.724)$, $Q T c$ interval $(P=0.737)$, and heart rate $(P=0.267)$. Overall, fewer CNS symptoms were reported for levobupivacaine than ropivacaine (218 versus 277). This study found that levobupivacaine and ropivacaine produce similar CNS and cardiovascular effects when infused IV at equal concentrations, milligram doses, and infusion rates. This study compared directly, for the first time, the toxicity of levobupivacaine and ropivacaine in healthy volunteers. Levobupivacaine and ropivacaine produced similar central nervous system and cardiovascular effects when infused IV at equal concentrations, milligram doses, and infusion rates Continuous interscalene brachial plexus block with a single dose of $0.75 \%$ bupivacaine $(150-210 \mathrm{mg}$ ) with adrenaline, continued with an infusion of plain $0.25 \%$ bupivacaine 0.25 
$\mathrm{mg} / \mathrm{kg} / \mathrm{h}$, was performed on 20 patients to provide analgesia during shoulder surgery and in the postoperative period. The control group included 20 patients who were given general anaesthesia for surgery after starting a continuous interscalene brachial plexus block; test dose of $0.75 \%$ bupivacaine $(22.5 \mathrm{mg})$ with adrenaline, continued with an infusion of $0.25 \%$ bupivacaine $0.25 \mathrm{mg} / \mathrm{kg} / \mathrm{h}$. Surgery was performed successfully under regional anaesthesia in 16/20 patients; $4 / 16$ were given one dose of fentanyl during the surgery, and diazepam or midazolam as supplementary sedation were given in 13/16 cases. For postoperative analgesia 35/40 patients had a fully functioning catheter for 20-26 hours and the need for oxycodone i.m. during that time was $1.5+/-0.4$ doses after regional anaesthesia $(n=14)$ and $1.8+/-0.4$ doses after general anaesthesia $(n=18)$. There was a statistically significant difference in the mean plasma bupivacaine concentrations between the groups, concentrations in the regional anaesthesia group being higher at 5, 30, $60 \mathrm{~min}$ and $3 \mathrm{~h}$ (maximum $2.3 \mathrm{micrograms} / \mathrm{ml}$ at $60 \mathrm{~min}$ ), but there was no difference between the values at $24 \mathrm{~h}$. One infusion of local anaesthetic was discontinued because of probable treatment-related side-effects (breathing difficulties, nausea). Mild local anaesthetic toxicity (dizziness, tinnitus) was noticed in four patients [7].

The acute central nervous and cardiovascular effects of the local anesthetics ropivacaine and bupivacaine were compared in 12 volunteers in a randomized double-blind manner with use of intravenous infusions at a rate of $10 \mathrm{mg} /$ $\mathrm{min}$ up to a maximal dose of $150 \mathrm{mg}$. The volunteers were all healthy men. They were familiarized with the central nervous system (CNS) toxic effects of local anesthetics by receiving a preliminary intravenous injection of lidocaine. The infusions of ropivacaine and bupivacaine were given not less than 7 days apart. CNS toxicity was identified by the CNS symptoms and the volunteers were told to request that the infusion be stopped when they felt definite but not severe symptoms of toxicity such as numbness of the mouth, lightheadedness, and tinnitus. In the absence of definite symptoms, the infusion was stopped after $150 \mathrm{mg}$ had been given. Cardiovascular system (CVS) changes in conductivity and myocardial contractility were monitored using an interpretive electrocardiograph (which measured PR interval, QRS duration, and QT interval corrected for heart rate) and echocardiography (which measured left ventricular dimensions from which stroke volume and ejection fraction were calculated). Ropivacaine caused less CNS symptoms and was at least $25 \%$ less toxic than bupivacaine in regard to the dose tolerated. Both drugs increased heart rate and arterial pressure. Stroke volume and ejection fraction were reduced. There was no change in cardiac output. Although both drugs caused evidence of depression of conductivity and contractility, these appeared at lower dosage and lower plasma concentrations with bupivacaine than with ropivacaine [8].

A total of 50 young soldiers hospitalized for high frequency hearing loss and tinnitus following exposure to gun impulse noise was studied in order to ascertain the effects of two kinds of medical treatment. A first group (18 subjects) was treated for 10 consecutive days with cerebral gangliosides. In a second group (17 subjects) gangliosides were associated with subcutaneous infiltration of bupivacaine chlorhydrate (0.5\%). A third group (15 subjects) was taken as control. An improvement in hearing threshold (= greater than $20 \mathrm{~dB}$ at 4-8 kHz) and a consistent relief of tinnitus was respectively found in $52 \%$ and $66 \%$ of the treated subjects, while hearing status and tinnitus persisted unchanged among the control group subjects. The amount of hearing improvement over the control group proved to be statistically significant, although no significant difference was demonstrated between the two kinds of medical treatment. Since therapy was initiated 5 to 21 days after acoustic trauma, these results indicate that a pharmacological treatment may be effective even in cases where diagnosis is forwarded relatively late in respect to the trauma [9].

Propranolol reduces the clearance of lidocaine by both reducing hepatic blood flow and inhibiting lidocaine metabolism. Bowdle et al., [10] investigated the possibility that propranolol reduces the clearance of bupivacaine as well. Bupivacaine, 30-50 mg, was administered intravenously to six normal human volunteers, over 10-15 min on two occasions, at least 2 weeks apart. Propranolol, $40 \mathrm{mg}$ orally every $6 \mathrm{~h}$, was used on one occasion, beginning $24 \mathrm{~h}$ prior to the bupivacaine administration. The sequence of the sessions was randomized. Twenty-two venous blood samples were obtained over 36 $\mathrm{h}$ in order to determine bupivacaine clearance, terminal elimination rate constant, and volume of distribution. All subjects experienced mild CNS toxicity, consisting of tinnitus, facial tingling, or subtle visual disturbances, associated with peak venous plasma concentrations of 0.81 to 2.7 micrograms/ $\mathrm{ml}$. Mean bupivacaine clearance was $0.33+/-0.12 \mathrm{l} / \mathrm{min}$ for the control session and $0.21+/-0.12 \mathrm{l} / \mathrm{min}$ during propranolol use, a significant $35 \%$ reduction ( $P$ less than 0.01 ). The terminal elimination rate constant (beta) was $0.27+/-0.16 \mathrm{~h}-1$ for the control session and $0.14+/-0.069 \mathrm{~h}-1$ with propranolol ( $\mathrm{P}$ less than 0.05); terminal elimination half-lives were 2.6 and $4.9 \mathrm{~h}$, respectively. Volume of distribution was unchanged. Because bupivacaine clearance should be relatively insensitive to hepatic perfusion, it appeared that propranolol caused a substantial inhibition of bupivacaine metabolism at the level of the hepatocyte. These data suggest that concomitant use of propranolol could result in the accumulation of a toxic concentration of bupivacaine.

\section{Intratympanic bupivacaine to relieve tinnitus}

A recent review by Meyer [11] in 2013 states that since the 1940s, various attempts have been made to treat peripheral tinnitus by way of intratympanic injection. This administration procedure requires only low concentrations of medication, thanks to the highly targeted delivery to the site of action 
and comes with minimal systemic exposure. While different compounds have been tested for their effects on tinnitus by intratympanic injection, there has been no breakthrough so far. Accordingly, the clinical use of intratympanic tinnitus treatments has remained limited to date. A more widespread adoption of this approach will require the development of specific medications for peripheral tinnitus, as well as proof of safety and efficacy, which would be determined from randomized controlled clinical trials.

Currently there are 5364 articles on Pubmed related to Tinnitus treatment. None of it mentions the use of Intratympanic bupivacaine instillation. It is the first time that such treatment is suggested. Clinical studies should be done in order to evaluate this new treatment modality.

\section{Competing interests}

The author declare that he has no competing interests.

\section{Publication history}

Received: 21-Apr-2013 Revised: 17-May-2013

Accepted: 21-May-2013 Published: 24-May-2013

\section{References}

1. Claus-Frenz Claussen: Chemically Induced or Drug Induced Tinnitus. The International Tinnitus Journal 1996, 2:1-2. I Website

2. Dippenaar JM: Local anaesthetic toxicity. Southern African Journal of Anaesthesia and Analgesia 2007, 13(3):23-28. | Article

3. Eldor J, Frankel DZ, Olshwang D and Davison JT: Tinnitus as an early diagnostic sign of inadvertent intravenous injection of bupivacaine epidural test dose. Resuscitation 1988, 16:303-4. | PubMed

4. Weinmeister KP: Prolonged suppression of tinnitus after peripheral nerve block using bupivacaine and lidocaine. Reg Anesth Pain Med 2000, 25:67-8. | Article | PubMed

5. Weinmeister KP: Prolonged suppression of tinnitus after peripheral nerve block using bupivacaine and lidocaine. Reg Anesth Pain Med 2000, 25:67-8. | Article | PubMed

6. Stewart J, Kellett $N$ and Castro $D$ : The central nervous system and cardiovascular effects of levobupivacaine and ropivacaine in healthy volunteers. Anesth Analg 2003, 97:412-6, table of contents. | Article | PubMed

7. Haasio J, Tuominen M and Rosenberg PH: Continuous interscalene brachial plexus block during and after shoulder surgery. Ann Chir Gynaecol 1990, 79:103-7. | PubMed

8. Scott DB, Lee A, Fagan D, Bowler GM, Bloomfield P and Lundh R: Acute toxicity of ropivacaine compared with that of bupivacaine. Anesth Analg 1989, 69:563-9. | Article | PubMed

9. Blotta P, Turrini M, Pastore A, Prosser S, Narne S and Piantoni S: [New therapeutic prospects in acute acoustic trauma]. Acta Otorhinolaryngol Ital 1989, 9:349-55. | PubMed

10. Bowdle TA, Freund PR and Slattery JT: Propranolol reduces bupivacaine clearance. Anesthesiology 1987, 66:36-8. | Article | PubMed

11. Meyer T: Intratympanic treatment for tinnitus: A review. Noise Health 2013, 15:83-90. | Article | PubMed

\section{Citation:}

Eldor J: Intratympanic bupivacaine instillation for tinnitus treatment? journal of Anesthesiology and Clinical Science 2013, 2:23.

http://dx.doi.org/10.7243/2049-9752-2-23 